\title{
Digitizing the Calibration Process
}

\author{
Peter Sherwin ${ }^{1 *}$ \\ ${ }^{1}$ Eurotherm by Schneider Electric, EOS Eurotherm Online Services, Faraday Close, Worthing. UK.
}

\begin{abstract}
Industry professionals understand that risk is inevitable; it can be managed but not eliminated. Managing risk is not easy, especially with today's products manufactured through complex and multi-tiered supply chains. Compliance and Calibration activities are used to aid management of risk in Industrial processes. Given the importance of this activity; it is necessary to ensure that any calibration undertaken is done accurately, records are held for fast retrieval, and the activity is carried out in the most cost effective manner. The most efficient method of managing the calibration process is to make use of modern digital tools.
\end{abstract}

\section{Introduction}

Regular comparison of an instrument to a standard (calibration) enables higher confidence and less risk in the accuracy of the process under check. Neglecting these calibration activities can result in unscheduled production downtime, product and process issues and potential product recalls and rework. In the worst case; a drift in a sensor unchecked over a period time (when situated in a hazardous area) can risk employee safety.

\section{Common Calibration Systems}

\subsection{Paper-based Calibration Certificates}

Engineers and Technicians conduct calibration on field and laboratory based instrumentation and compare against a set standard. The 'as-found' (when appropriate) and 'as-left' results are typically written down in laboratory books, on printed sheets or notepads and then ultimately transcribed to produce a certificate of calibration. The end document might be produced through common PC-based tools (Word processing or Spreadsheet based programs) or even entered into a customized calibration database before the certificate is produced.

The action of transferring information from a handwritten page to a final certificate is subject to human error in transcription as well as adding significant administration time and cost to create the final certificate. Depending on the engineers schedule, it can take days or even weeks before the instrument owner receives their calibration reports from a Service providers or Internal Calibration team.

There are also significant concerns with creating and storing a printed document:
- Storage in a single physical location - requires time and resource to access.

- Offsite storage is often used to hold multiple years of records (up to 30 years of past records are required for certain industries). This lengthens the time and cost required for access.

- Filing is prone to human error. The time to retrieve a misfiled document can add hours or even days to a search.

- Damage by frequent handling can quickly reduce the life of paper documents.

- Paper records are also vulnerable to other risks; a site disaster can lead to loss of documents through fire or water damage.

\subsection{Digitizing the Calibration Process}

There are a number of methods to digitizing the calibration processes and avoid the pitfalls of a manual system. Popular generic PC programs, Desktop Calibration Software and ERP modules are discussed below.

\subsubsection{Word processing or Spreadsheet Programs}

Many companies still use spreadsheet or wordprocessing software to capture their calibration results and print paper copies of calibration certificates or supply pdf versions of the results to the instrument owner. This is a relatively inexpensive way to digitize the calibration process but does usually require double entry of data (quick pen/paper record before entry into the laptop/desktop).

Corresponding author: peter.sherwin@schneider-electric.com

C The Authors, published by EDP Sciences. This is an open access article distributed under the terms of the Creative Commons Attribution License 4.0 (http://creativecommons.org/licenses/by/4.0/). 


\subsubsection{Desktop Calibration Software}

Desktop software is traditional software that is installed on a desktop or laptop computer. The files are stored locally or on a shared network drive with the processing taking place on the local computer. The majority of commercially available calibration management systems are still desktop based. Early customized systems started life in the 1980's and have evolved into expansive databases with rich feature sets. Some improvement in the User Interface Design has taken place to aid data entry and simplify training requirements. These systems can offer a richer calibration experience when processes and procedures are also covered by the system.

Certain software providers have also attempted to combine other applications such as Maintenance requirements to be able to save time, reduce training, combine key operational processes, reduce costs and increase productivity.

\subsubsection{ERP Based Systems}

Major providers of ERP systems also offer modules for asset management, maintenance and calibration. Unfortunately these modules tend to be expensive and can either be overly complex or alternatively only offer fixed basic features. Customization to specific end-user requirements comes at a cost.

\section{Modern Digital Tools}

To provide the best value from a digitized calibration process, it is important to address the deficiencies of a paper-based system and avoid the potential data double entry associated with Spreadsheet-based solutions. Some form of user configuration of the calibration process also avoids the expensive customization requirements often associated with ERP modules. A good set of rules for digital systems is outlined below:

- Enter data directly into a mobile device (smartphone, tablet, portable laptop etc.).

- Only store a virtual copy of the certificate for authorized access from anywhere/anyone

- Document attributes should enable quick search and retrieval.

- Virtual storage should assure long-term backup.

- Cloud-based storage with disaster-proof backup is ideal.

- Methods to customize the calibration process using a configurable workflow.

On a practical point not all Industrial sites offer a robust Wi-Fi connection or have a stable phone signal. To be able to provide one-off data entry solutions using any smart device, the mobile device should be able to operate in both offline and online mode. This is best achieved by using a dedicated app (see The App Economy).

\subsection{The App Economy}

10 years ago virtually no mobile apps existed, yet today there are over 90 billion app downloads. The app economy is booming and made popular because apps are an efficient and simple way to help people accomplish tasks in an easy and less time consuming manner. Initially dedicated solely to the consumer world, the app world has evolved to offer many business apps. Popular business apps include; team communication, task, time and project management, payment and accounting apps and Sales and Customer Relationship Management.

More recently, dedicated calibration apps (example: $\mathrm{eCAT}^{\mathrm{TM}}$ Calibration Management Tool [1]) have been developed to simplify and increase the efficiency of calibration activities.

\subsubsection{App-based Calibration Solutions}

Multi-tiered supply chains and networks have spawned a complex arrangement of partners, suppliers and customers. Enabling visibility, scheduling and update of calibration activities across a range of multiple service providers and internal engineer resources requires a digital process that can cope with multi-tenant secure access. Fortunately, this is now possible by leveraging recent advances in cloud-based technology and appdesign.

Fixed format database design does not provide sufficient flexibility in data entry to aid the user to undertake the correct calibration when dealing with multiple complex industry standards. The latest apps have developed specific customizable workflow technology to enable setups to be created through the web-based portal and downloaded to the app to provide the engineer with stepby-step prompts to undertake the correct calibration process.

When the calibration record is digitized, instant authorized access is opened up to multi-users across the company regardless of location. This enables the production department to be aware of calibration schedules and minimize disruption to production. By also capturing descriptive data about each document, retrieval of relevant information is fast and efficient. Leveraging app-based technology has also enabled locally printed QR Code labels to link directly to the completed Calibration Certificate. This direct access to the certificate has enabled customers to effortlessly meet audit requirements.

The Certificate attributes such as Date and time of calibration, next due date, engineer, location etc. enable the systems to be able to schedule and plan and alert when the next calibration is due. 


\section{Summary}

Digital offers, disruptive technologies and the current political climate are increasing the rate of change across Industry. Managing risk within this fluid and everchanging environment is complicated. To navigate this change successfully requires the best tools and techniques to avoid costly, unwieldy and inefficient manual processes. It is time to digitize your calibration process!

\section{References}

1. eCAT; http://www.eurotherm.co.uk/eos webpage accessed 20Jun17 (2017) 